\title{
RoboBat: Dynamics and Control of a Robotic Bat Flapping Flying Testbed
}

\author{
P. Daniel Kuang *, Michael Dorothy ${ }^{\dagger}$, and Soon-Jo Chung ${ }^{\ddagger}$
}

\begin{abstract}
This paper investigates the control of the phase difference in between three different motions of bat flight: pitching, flapping, and lead-lag. For active control, a robotic bat test bed capable of simulating different wing motions is used to test the control of these wing motions and the phase differences using central pattern generators (CPG's). Previous work with the robotic bat is expanded upon by modifying the robotic bat test bed to allow for three dimensional motions of the entire bat, instead of only the wings. This is done by mounting the robotic bat onto a $3 \mathrm{D}$ pendulum. Experiments analyzing the steady state behavior of the bat's flight with varying phase differences showed a change of pitch while elevation and forward velocity remains constant. This shows promising results regarding the relation between phase differences of wing motions and longitudinal stability.
\end{abstract}

\section{Nomenclature}

$\phi_{w}, \psi_{w}, \theta_{w}$

$\mathbf{x}_{i}=\left(u_{i}, v_{i}\right)^{T}$

$\mathbf{f}\left(\mathbf{x}_{i} ; \rho_{i}\right)$

$\rho_{i}$

$\lambda$

$\omega$

$a_{i}$

$\sigma$

$\mathbf{R}\left(\Delta_{i j}\right)$

$\Delta_{i j}$

$n$

$k$

Subscript

$i$

$R, L$
Flapping, lead-lag, and pitch angles of each wing (left, right)

State vector of the $i$-th Hopf oscillator

Hopf nonlinear equations in the vector form with radus $\rho_{i}$

Radius of the limit cycle from the $i$-th Hopf oscillator

Common rate of convergence of Hopf oscillators

Common oscillation frequency of Hopf oscillators, rad/s

Amplitude bias of the $i$-th Hopf oscillator

Bifurcation parameter. $\sigma=1$ for a stable limit cycle or $\sigma=-1$ for convergence to $a_{i}$. $2 \times 2$ rotational transformation matrix

Phase lead of the $i$-th Hopf oscillator from the $j$-th

Total number of Hopf oscillators in the CPG network

Coupling gain of the coupled Hopf oscillators

Variable number of the coupled Hopf nonlinear oscillators

Right or left wing

\section{Introduction}

Animals such as birds, bats and insects are capable of agile flight motions and rely mostly on flapping their wings for stability and control. The relatively small size of these animals limits their flight regime to a Reynolds number on the order of $10^{4}-10^{5}$. At such low Reynolds numbers the efficiency of fixed wings drops significantly due to unsteady aerodynamic effects. Thus a main goal of this project is to emulate and adapt these methods of flight, which have been time-tested over millions of years, to micro aerial vehicles (MAV's). Applications for more agile MAV's include surveillance, reconnaissance, and monitoring of infrastructures.

The design of flapping flight micro aerial vehicles presents numerous control and dynamic challenges. Some previous examples of flapping flight models can be found in [1], [2], [3], [4], [5], [6], and [7], as well as in commercial products. All of these systems use a crankshaft mechanism to produce the flapping motions, and

\footnotetext{
*Master's Student, Department of Aerospace Engineering, pdkuang2@illinois.edu. Student Member AIAA.

$\dagger$ Doctoral Student, Department of Aerospace Engineering, dorothy1@illinois.edu. Student Member AIAA.

${ }^{\ddagger}$ Assistant Professor, Department of Aerospace Engineering, sjchung@illinois.edu. Senior Member AIAA.
} 
are therfore limited to producing sinusoidal motions of a fixed amplitude with variable frequency. However, experiments with high speed cameras have shown that the flapping motions in bats are not sinusoidal. ${ }^{8}$ Furthermore, several parameters of the flapping motion change depending on flight conditions. The flapping amplitude varies, along with the phase difference between different wing motions, the wing beat frequency and the angle of attack. Studies of insect flight such as in [1] and [2] accurately model insect flight by allowing changes in pitch and stroke plane angle. However, these systems do not allow changes in stroke amplitude, and there is no ability to generate arbitrary stroke motions.

While ornithopters capable of flapping their wings in a single direction have existed for some time, in nature animals flap their wings in several complex movements in three dimensions. Animals capable of flight utilize several, different complex three dimensional wing motions such as flapping, pitching and a lead-lag motion. These motions must be understood and controlled in order to achieve fully autonomous flapping flight. In this paper, a test bed modeled after a bat is used to simulate flapping, pitching and lead-lag motions. The wing motions are controlled by central pattern generators (CPG's). ${ }^{9}$

The next goal was to design and engineer a flapping flight test bed capable of moving in three-dimensions, as opposed to remaining static on a stand. This test bed can be used to verify previous research regarding the synchronization and control of phase differences between wing motions to achieve stability. ${ }^{9}$ Using the base of a Quanser 3DOF Helicopter, the rotors of the helicopter were replaced with the robotic bat itself. This paper describes the current test bed built for the robotic bat and the experiments that can be conducted with it.

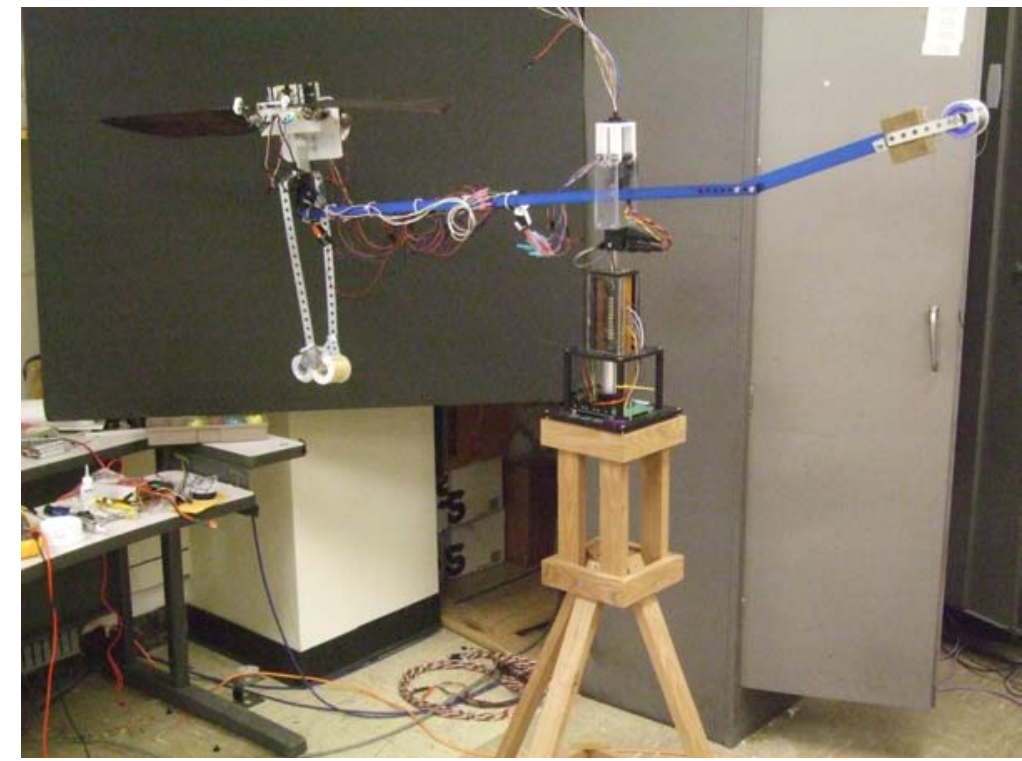

Figure 1. Test bed overview

\section{Biological Inspiration}

Flapping flight has historically been inspired from nature; birds in flight formed the basis of all early attempts at flight. Therefore there are several important examples of flight in nature that we derive our design from. Insects have been a popular choice because of the relatively simple configuration of their flight system. They require only two control inputs (stroke angle and pitch angle) which can be modeled as a sinusoid. Control design is much easier, as averaging methods are valid within the high frequencies in insect flight. Unfortunately, the aerodynamics of insect flight vary significantly from the mechanics of bat flight and bird flight. Unsteady effects dominate their flight regime because of the extremely low Reynolds number. Insect wings are typically very simple and mostly rigid. ${ }^{10}$

In bird flight, the wings and flight become more complex. Light weight bone structures in the wing, the complex airfoils formed by the feathers, and the addition of the elbow and wrist joints make birds more difficult to simulate. Their flight mechanics also differ significantly from insects as well.

Bat flight differs from both bird and insect flight. They operate in a Reynolds number range where 
unsteady effects are important at low speeds but decreasingly important at the higher range of flight speeds. Because bats are equally or even more complicated than birds, and because of their flight performance, we have chosen to model our robotic test bed after a bat. Bat flight is also well suited to Central Pattern Generator control because it relies heavily on the synchronization of phase differences between several different oscillatory motions.

\section{II.A. Central Pattern Generators}

A principle factor in choosing how many degrees of freedom were necessary for the test bed was based on biological principles. Another biological principle followed in the design of the bat was choosing the control scheme to be used. Many creatures produce their motion by synchronizing periodic motions of limbs, such as running, swimming or flapping. They do this by coupling biological oscillators and synchronizing their outputs. Biological oscillators rely on short timescale (ms) neuron dynamics including spike-bursting, spike frequency adaptation, and post-inhibitory rebound. Herrero-Carrón, et. al. ${ }^{11}$ designed a control law for modular robots by approximating short timescale neuron dynamics. Because there is such a short timescale required for integration, the neuron dynamics were integrated offline. We are unlikely to be able to perform such strict mimicry in an online controller as we add additional neurons for feedback, active control of phase differences, or gait transitions.

In order to make online control more feasible, we can emulate these biological oscillators by using limit cycle oscillators coupled together. A limit cycle oscillator is a nonlinear model that converges to a stable trajectory which is called the limit cycle. Because of this convergence the oscillator will quickly forget disturbances and converge back to the stable limit cycle. If the oscillator itself is a smooth vector field, we can smoothly transition between desired trajectories without abrupt changes being required in the motor output.

Following Chung and Dorothy, ${ }^{9}$ we use the following limit-cycle model called the Hopf oscillator, named after the supercritical Hopf bifurcation model with $\sigma=1$ :

$$
\begin{array}{r}
\frac{d}{d t}\left(\begin{array}{c}
u-a \\
v
\end{array}\right)= \\
{\left[\begin{array}{cc}
-\lambda\left(\frac{(u-a)^{2}+v^{2}}{\rho^{2}}-\sigma\right) & -\omega(t) \\
\omega(t) & -\lambda\left(\frac{(u-a)^{2}+v^{2}}{\rho^{2}}-\sigma\right)
\end{array}\right]\left(\begin{array}{c}
u-a \\
v
\end{array}\right)+\mathbf{u}(t)} \\
\text { Equivalently, } \quad \dot{\mathbf{x}}=\mathbf{f}(\mathbf{x} ; \rho ; \sigma)+\mathbf{u}(t), \text { with } \quad \mathbf{x}=(u-a, v)^{T}
\end{array}
$$

where the $\lambda>0$ denotes the convergence rate to the symmetric limit circle of the radius $\rho>0$ and $\mathbf{u}(t)$ is an external or coupling input. In that work, it was shown that coupled networks of Hopf oscillators on balanced graphs exhibit smooth exponentially stable behavior in both oscillatory mode and fixed point mode.

The possibly time-varying parameter $\omega(t)>0$ determines the oscillation frequency of the limit cycle. A time-varying $a(t)$ sets the bias to the limit cycle such that it converges to $u(t)=\rho \cos (\omega t+\delta)+a$ and $v(t)=\rho \sin (\omega t+\delta)$ on a circle. The output variable to generate the desired oscillatory motion of each joint is the first state $u$ from the Hopf oscillator model in Eq. (1).

Synchronization means an exact match of the scaled amplitude or the frequency in this paper. Hence, phase synchronization permits different actuators to oscillate at the same frequency but with a prescribed phase lag. In essence, each CPG dynamic model in Eq. (1) is responsible for generating the limiting oscillatory behavior of a corresponding joint, and the diffusive coupling among CPGs reinforces phase synchronization. For example, the flapping angle has roughly a 90-degree phase difference with the pitching joint to maintain the positive angle of attack (see the actual data from birds in [10]). The oscillators are connected through diffusive couplings, and the $i$-th Hopf oscillator can be rewritten with a diffusive coupling with the phase-rotated neighbor.

$$
\dot{\mathbf{x}}_{i}=\mathbf{f}\left(\mathbf{x}_{i} ; \rho_{i}\right)-k \sum_{j \in \mathcal{N}_{i}}^{m_{i}}\left(\mathbf{x}_{i}-\frac{\rho_{i}}{\rho_{j}} \mathbf{R}\left(\Delta_{i j}\right) \mathbf{x}_{j}\right)
$$

where the Hopf oscillator dynamics $\mathbf{f}\left(\mathbf{x}_{i} ; \rho_{i}\right)$ with $\sigma=1$ is defined in Eq. (1), $\mathcal{N}_{i}$ denotes the set that contains only the local neighbors of the $i$-th Hopf oscillator, and $m_{i}$ is the number of the neighbors. The $2 \times 2$ matrix $\mathbf{R}\left(\Delta_{i j}\right)$ is a 2-D rotational transformation of the phase difference $\Delta_{i j}$ between the $i$-th and $j$-th oscillators. The positive (or negative) $\Delta_{i j}$ indicates how much phase the $i$-th member leads (or lags) from the $j$-th member and $\Delta_{i j}=-\Delta_{j i}$. The positive scalar $k$ denotes the coupling gain. 


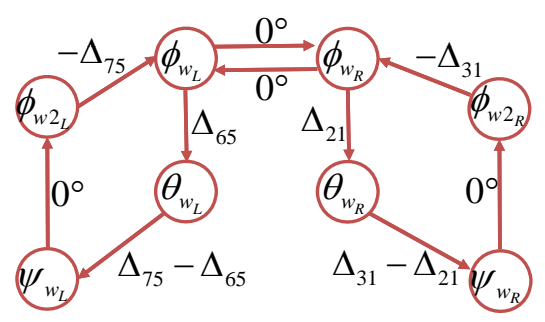

(a) Configuration A

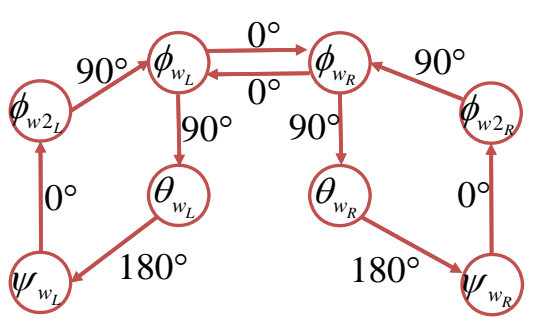

(b) Symmetric Configuration A

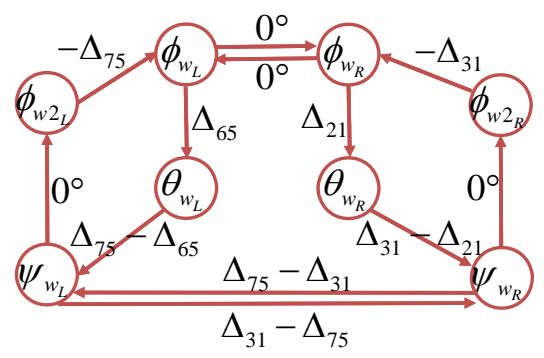

(c) Configuration B

Figure 2. Graph configurations of the coupled Hopf oscillators on balanced graphs. Many other configurations are permitted in this paper and the unidirectional couplings can be replaced by the bi-directional couplings. The numbers next to the arrows indicate the phase shift $\Delta_{i j}$ from the $i$-th member to the $j$-th member while Figure $\mathbf{b}$ shows the nominal values of the phase shift from the symmetric wing configuration such that $\Delta_{21}=\Delta_{65}=90$ deg. and $\Delta_{31}=\Delta_{75}=-90$ deg. Such phase shifts define flight modes (wing movement gaits).

Numerous configurations are possible as long as they are on balanced graphs ${ }^{12}$ and we can choose either a bidirectional or a uni-directional coupling between the oscillators. The numbers next to the arrows indicate the phase shift $\Delta_{i j}$, hence $\Delta_{i j}>0$ indicates how much phase the $i$-th member leads. Since the graphs in Figure 2 are on balanced graphs, the number of input ports equal the number of output ports. Further, all the phase shifts $\left(\Delta_{i j}\right)$ along one cycle should add up to a modulo of $2 \pi$. Figure $2 \mathrm{~b}$ shows the nominal values of the phase shift from the symmetric wing configuration such that $\Delta_{21}=\Delta_{65}=90 \mathrm{deg}$. and $\Delta_{31}=\Delta_{75}=-90 \mathrm{deg}$.

\section{II.B. Control Design from Physical Intuition}

Chung, et. al., ${ }^{13}$ provided a simple intuition for phase difference control of longitudinal motion in flapping flight. With a zero bias lead-lag and a center of gravity coinciding with the stroke plane, a phase difference of 270 deg between the flapping CPG and the lead-lag CPG gives Azuma's ${ }^{10}$ elliptical model of flapping: negative lead-lag on downstroke, positive lead-lag on upstroke. The simplest analysis combines a maximum force with the most-negative lead-lag at the middle of the downstroke to predict a pitch-down moment on the body. Alternatively, if we see the phase difference to $90 \mathrm{deg}$, we see the maximum force coinciding with the maximum positive lead-lag at the middle of the downstroke, predicting a pitch-up moment. This intuition was confirmed in numerical simulations. In section IV, we test this intuition on the RoboBat.

\section{Experimental Setup}

\section{III.A. Design of Robotic Bat}

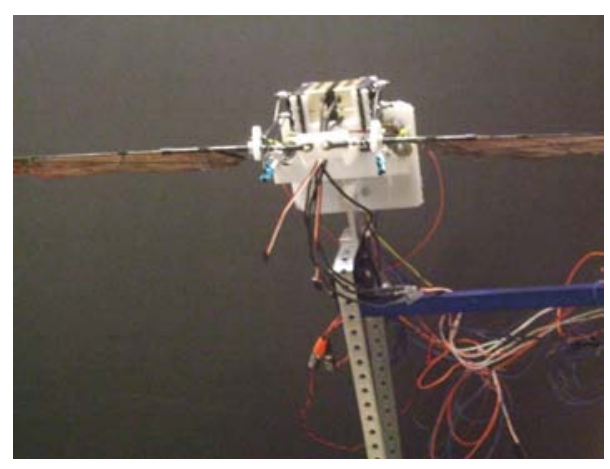

(a) Front View

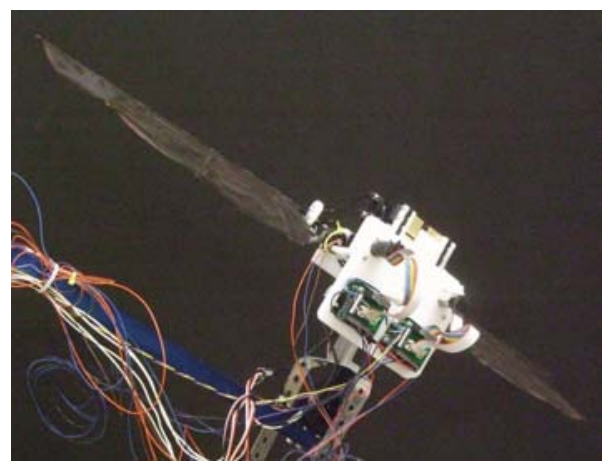

(b) Back View

Figure 3. Front and back views of robotic bat, mounted on Quanser 3DOF Helicopter stand

The robotic bat is a highly controllable platform, modeled after the kinematics of a bat. Eight degrees of freedom are provided; three in each shoulder joint and two for the amplitude of flapping. Shoulder joints are also analogous to human shoulder joints, able to move forward, backwards, up, down, and can twist in 
both directions. Those motions correspond to lead-lag, flapping, and pitching respectively. These 8 degrees

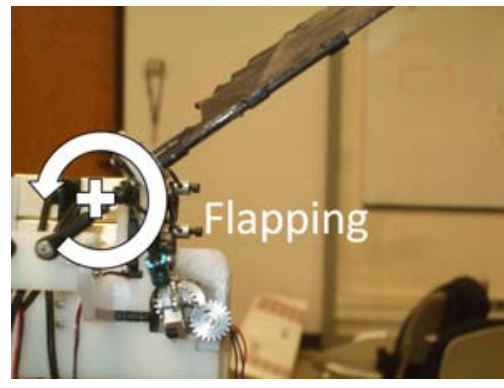

(a) Flapping Motion

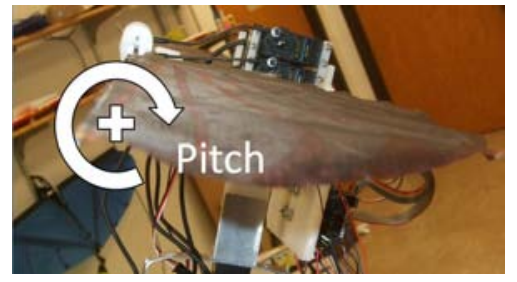

(b) Pitching Motion

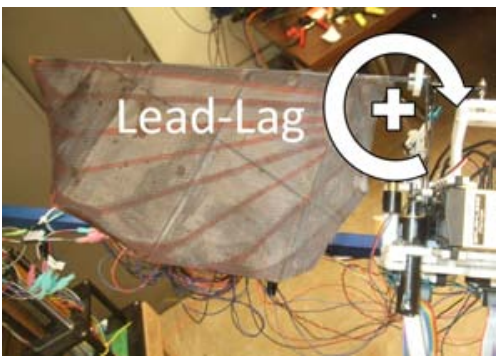

(c) Lead-Lag Motion

Figure 4. Positive wing motion directions

of freedom are combined with variable speed motors to allow for maximum flapping in control schemes. The flapping motion of the wings are independently powered by two 5 watt Maxon motors. Electronic controllers for the two Maxon motors allow for precise control of motor velocity and thus flapping frequency. All other degrees of freedom are controlled with Futaba servos. In the previous bat design from [9], the servos actuating the lead-lag and pitch motions were feather servos. The new structure uses bigger and more powerful servos for increased torque and speed. Two US Digital absolute encoders are attached to the sides of the two motors and connected with gears in order to measure the absolute position of the wings. This position data is used to create a closed loop controller for the wings and allows them to synchronize to a desired signal from the CPG's.

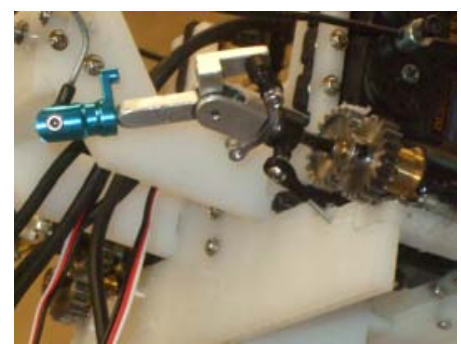

(a) Drive shaft to actuate flapping, with motor and encoder shown

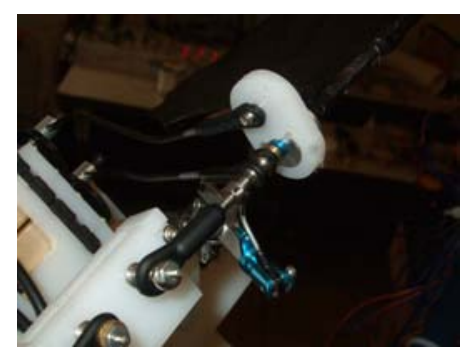

(b) Shoulder Joint, with pushrods for flapping, pitch, and lead-lag

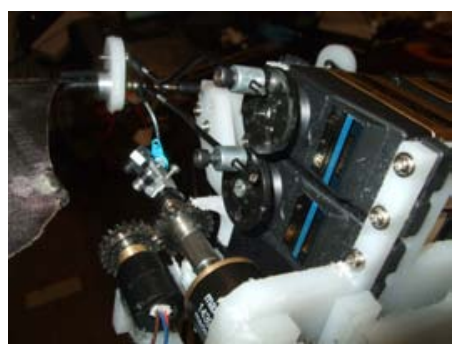

(c) Pitch (top) and lead-lag (bottom) servos

Figure 5. Close-ups of various components of robotic bat

The flapping amplitude is varied by a mechanism consisting of a moving crank arm and a rotating slider. A tail pitch plate used on RC helicopter tail rotors is used to control the flapping amplitude. As servo controls the slider on the tail pitch plate and moves it to vary the distance from the motor shaft to the crank arm. This changes the flapping amplitude. Additionally, the servo has to move only a small angular distance to change the flapping amplitude.

The main frame of the test bed was fabricated with a CNC machine. This method allows for quick changes in the design to be made. More complex parts can be machined. High density polyethylene was chosen as the main frame material for its low cost and ease of use with the CNC machine. All drive train materials are constructed of aluminum and steel, with non standard drive train parts being machined.

Controlling the robotic bat is done with a dSPACE DS1104 Controller board. The setup consists of a PPC board, which is mounted inside the computer via PCI slot, and a connector board which connects to the PPC board and provides an I/O interface for wiring to the robotic bat. The connector board outputs PWM signals and square waves to control the servos and motors, respectively. An analog/digital converter on the connector board allows the absolute motor encoders to output a voltage to the dSPACE board and have it converted to a digital signal, which can be read by the computer.

\section{III.B. Design of 3DOF Test Bed}

To hold the robotic bat in place while providing movement in three directions, the Quanser 3DOF Helicopter was used for its stand, encoders and Q4 board. The stand of the Quanser 3DOF Helicopter uses built-in 


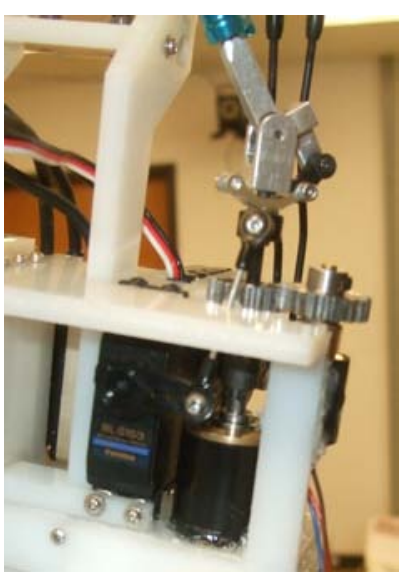

Figure 6. Close-up of amplitude controlling servo and drive shaft

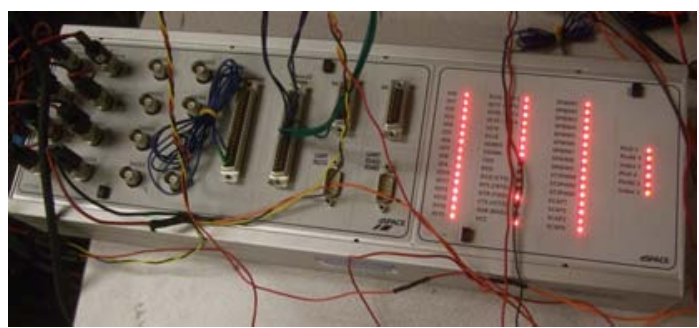

Figure 7. dSPACE connector board

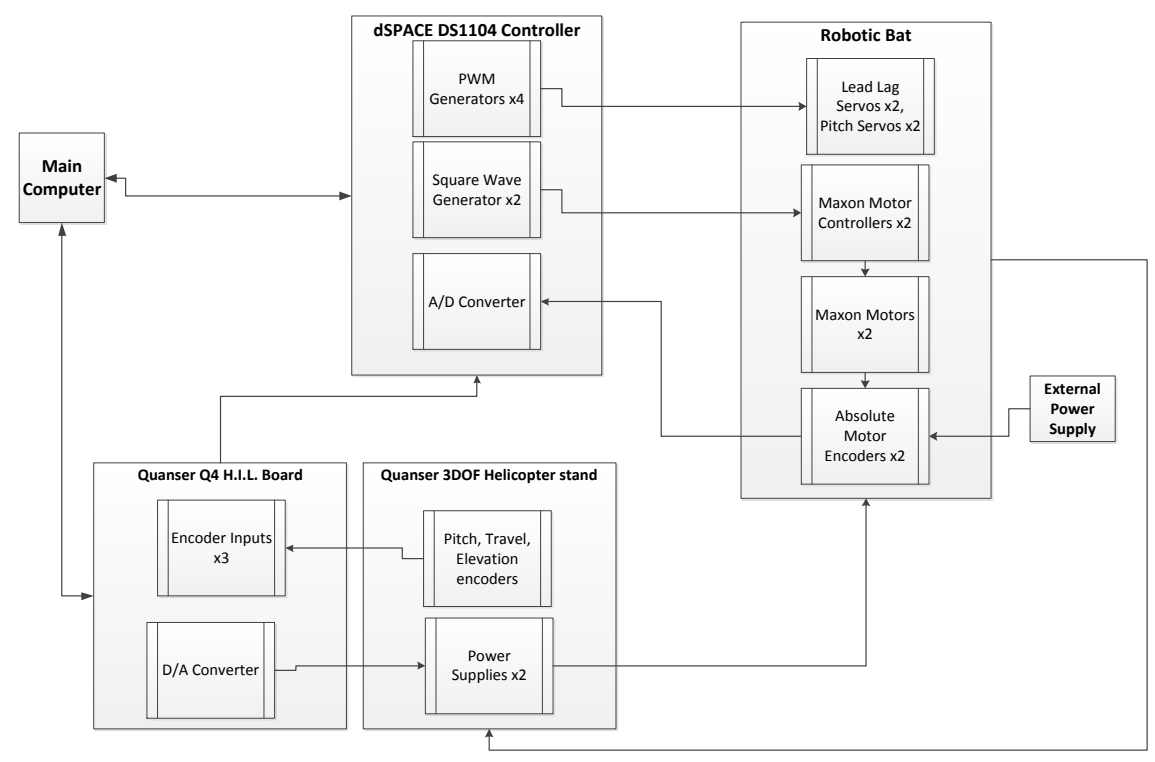

Figure 8. Schematic of test bed setup

encoders to measure pitch, travel, and elevation, which can be feed information regarding the robotic bat's position and orientation back into the controller of the robotic bat.

A wooden base was constructed to provide higher elevation of the stand and to provide a larger, more stable base for the entire test bed to rest upon. To power the robotic bat, the RCA connectors which were originally used to power the helicopter rotors included with the stand are used to provide the voltage necessary for the servos and motors of the bat. 
Electrical connections to the Quanser 3DOF Helicopter are controlled by the Quanser Q4 Hardware in the Loop (HIL) board. The Q4 board provides us with D/A voltage outputs and encoder inputs . ${ }^{14}$ Two $\mathrm{D} / \mathrm{A}$ voltage outputs on the Q4 board allow the necessary voltages needed for the servos and motors of the robotic bat. Three encoder inputs are used to read the encoder counts from the travel, pitch, and elevator encoders.

A constant electrical connection must be maintained between the controlling computer and the robotic bat so that controlling signals can be sent. dSPACE is used to create and send the controlling signals to the robotic bat, and since a wireless transfer method between dSPACE and the robotic bat's servos and motors was deemed too difficult if not infeasible, an Orbex Group slip ring was used to main a contact electrical connection while still allowing the stand to rotate freely. The wires from dSPACE were threaded through the ceiling of the laboratory to prevent physical interference with the rotation of the helicopter stand's arm. Care is taken so that the wires do not interfere with the motion of the bat.

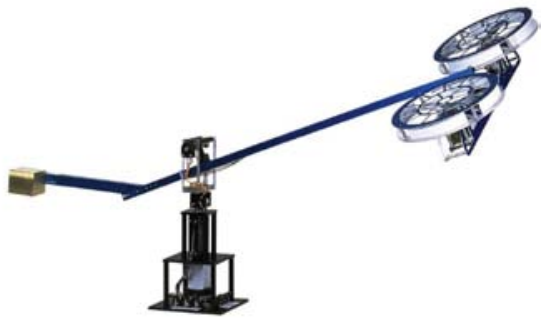

(a) Quanser 3DOF Helicopter ${ }^{15}$

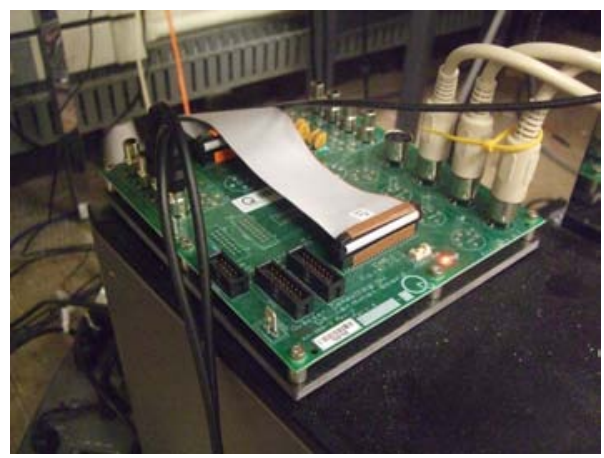

(c) Quanser Q4 board

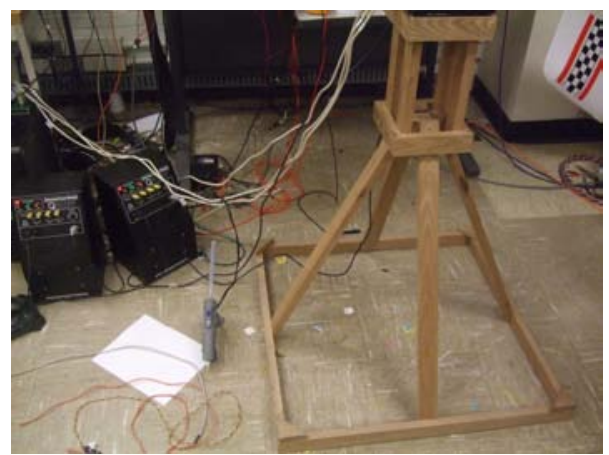

(b) Wooden stand to hold Quanser 3DOF Helicopter stand, with power sources to the left

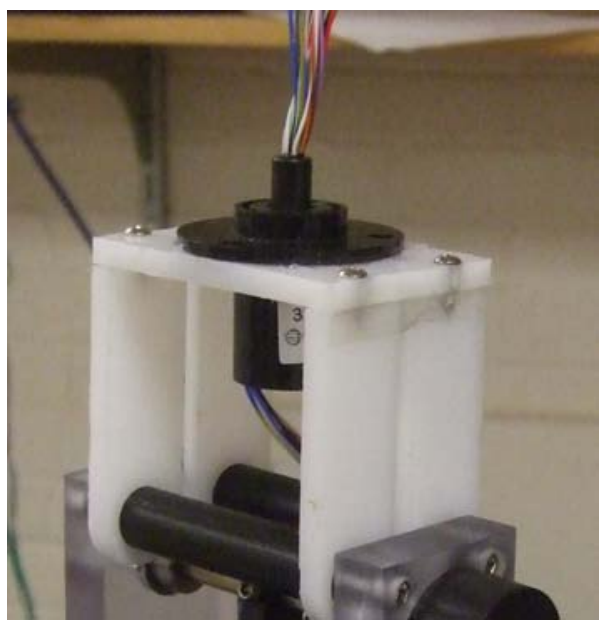

(d) Slip ring, mounted on top of stand and connected to dSPACE/computer and robotic bat

Figure 9. Components of test bed

The helicopter stand includes an elevation counterweight located at the opposite end of the helicopter stand's arm. The position of this weight can be changed to simulate different effective weights of the robotic bat. This weight brings the elevation axis closer to neutral stability and would simulate the low weight (on the order of 30 grams) of the average bat. ${ }^{16}$ To change the stability point of the robotic bat's pitch, a pitch counterweight is attached below the robotic bat. This pitch counterweight is added to the test bed by using perforated metal straps to attach it to a certain distance below the point of connection between the robotic bat and the helicopter stand's main rotating arm. The position of this pitch counterweight can be varied by moving the weight to different positions on the perforated metal straps, which would change the inherent longitudinal stability of the robotic bat. This allows us to test our control schemes on differing levels of natural stability. Counterweights were calibrated in order to bring the points of stability closer to neutral 
stability, with a slight amount of positive stability.

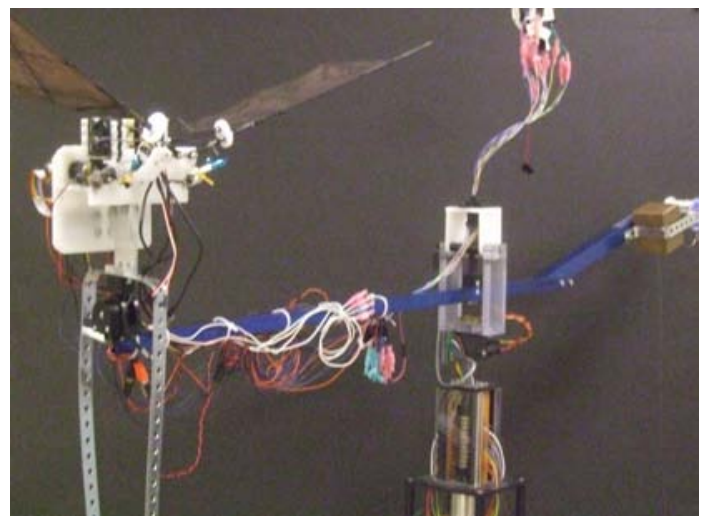

(a) Robotic bat mounted on horizontal arm of Quanser 3DOF Helicopter stand

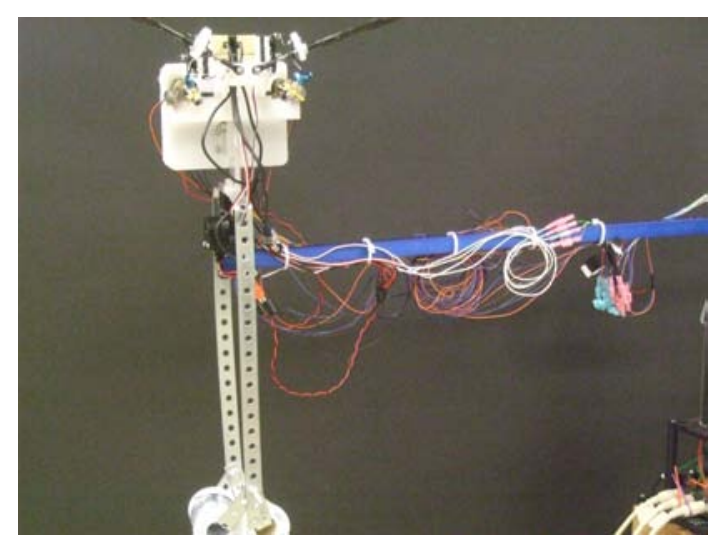

(b) Closer view of robotic bat, with pitch counterweight

Figure 10. View of test bed set up.

\section{III.C. Controller Design}

dSPACE, MATLAB, and Simulink are used to write the controller for the entire test bed. A Simulink model using dSPACE's own real time interface and blocksets was used in [9]. This model has since been improved to allow for better synchronization of motors to CPG signals, and more control over the phase differences between wing motions. To create a closed loop controller for the robotic bat with respect to its position and orientation on the Quanser 3DOF Helicopter stand, data must be exchanged between the dSPACE DS1104 and Quanser Q4 boards. In particular, the controller which is compiled and run on the DS1104 board requires the encoder data from the Quanser Q4 board. Quanser also includes its own real time interface and blockset for Simulink, however it is currently not possible to compile a Simulink model containing both dSPACE and Quanser blocks due to how they are compiled to their respective real time processor boards. Therefore, a different approach was taken to interface both Quanser and dSPACE so that data could be exchanged between the two boards.

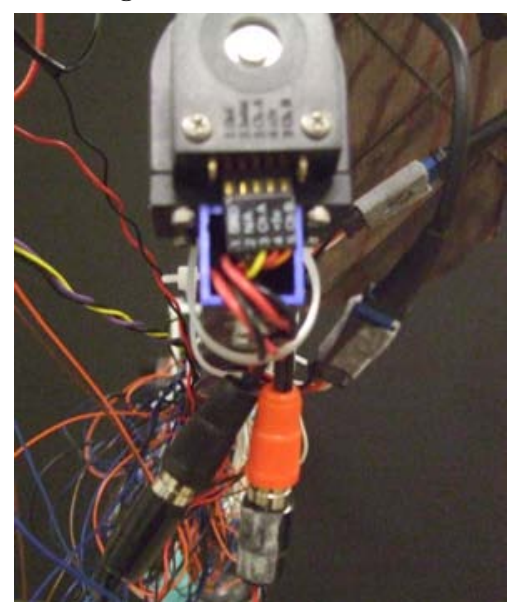

(a) Pitch encoder

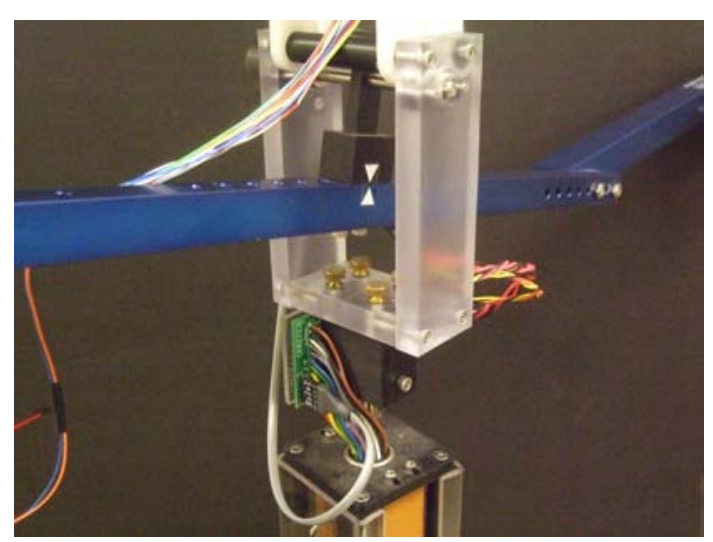

(b) Elevation and travel encoders

Figure 11. Encoders on Quanser 3DOF helicopter stand

In order for the robotic bat to receive encoder data from the Quanser 3DOF Helicopter's encoders, we designed a software interface using dSPACE's MLIB and Quanser's Stream API. MLIB is a library of MATLAB functions which allowed for communication with the DS1104 board, and Quanser's Stream API contains MATLAB functions for data transfer between the computer and the Q4 board. Raw encoder count data was read into MATLAB, which then converted this data into radians and degrees, and wrote this data 
into the robotic bat's Simulink experiment file. The DS1104 board ran the Simulink experiment in real time and thus we could get feedback on the robotic bat's attitude and orientation in real time via the encoders. This encoder data is used to develop a closed loop controller for the elevation, pitch, and travel angles and velocities for the bat. Ideally, the closed loop controller would use the encoder data as an input, and would change the phase differences between the pitch, flapping, and lead-lag motions as an output to control longitudinal modes.

The relatively high weight of the robotic bat, combined with the relatively high weight of the pitch counterweight on the opposite end of the pitching arm, create a considerable moment of inertia. Because of this moment of inertia, even a small amount of pitch variation due to the modulation of phase differences shows a significant effect with regards to maintaining longitudinal stability.

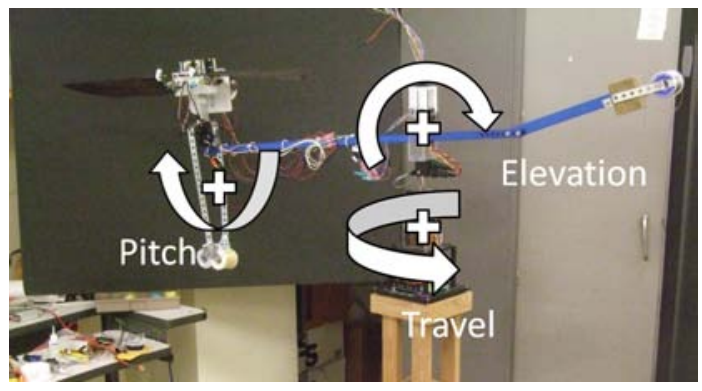

Figure 12. Test bed body motion directions

\section{Experimentation}

Experiments are conducted by commanding flapping frequency and phase differences while observing the bat's orientation and velocities from the encoder data. Using dSPACE's ControlDesk software, a GUI is created for direct interaction with the real time controller of the bat. Control variables can be changed and plotted in real time, and data is captured and saved to a MATLAB binary file.

As mentioned in Section IIIIII.B, there is an offset between the center of the bat and the pitch rotational point. This creates a coupling between the dynamics of the second pendulum with the longitudinal dynamics of the bat. While we use this to our advantage to obtain stability states desired for testing phase difference control, it necessarily creates a large rotational moment of inertia that is many times that of an actual bat. Therefore, we expect the pitching moments from phase difference control to have less effect in this experimentation than in free flight of a low moment of inertia bat. Regardless, we hope to see pitch control via only flapping/lead-lag phase difference even in this set-up.
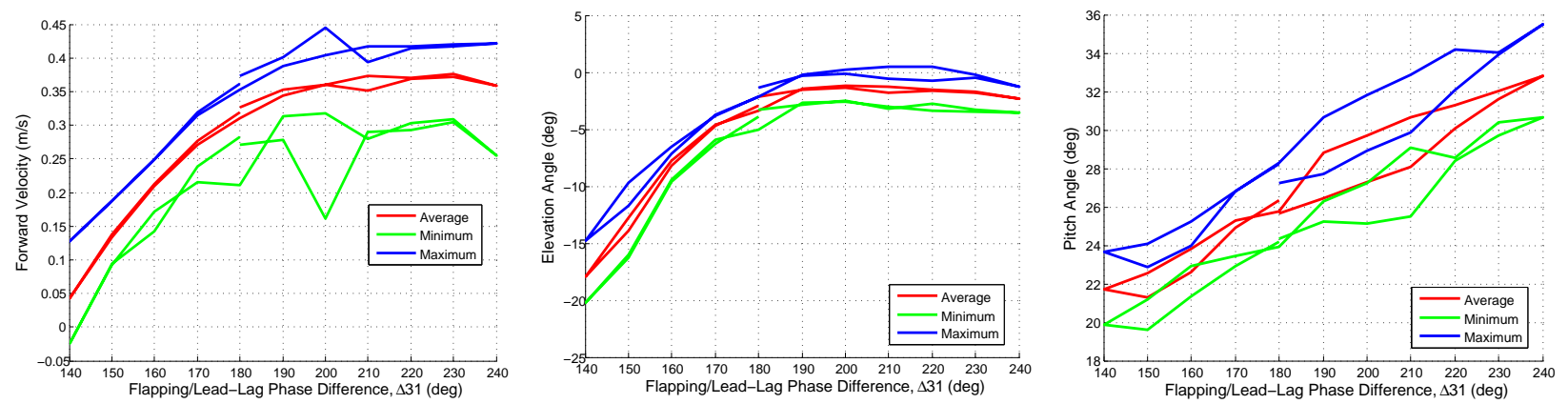

Figure 13. Open-loop pitch control via phase differences $(2 \mathrm{~Hz})$.

Two sets of experiments have been performed thus far. They have both focused on steady-state behavior of pitching motion with respect to phase differences. Most variables were held constant and are given in Table 1. In the first experiment, the phase difference between flapping and lead-lag, $\Delta_{31}$, was varied between $140 \mathrm{deg}$ and $240 \mathrm{deg}$ twice. The system was allowed to converge to a non-equilibrium steady state. Encoder data was captured for 20 seconds. Figure 13 shows the minimum, maximum, and average value over the 20 second period. As expected, between 180 and $240 \mathrm{deg}$, the forward velocity and elevation curves look 
very flat, but the pitch angle increased between 6 and 8 degrees. This corresponds with the idea that lift and thrust generation remained similar while only a control moment was created. It is postulated that a free flight system with a low pitching moment of inertia would see much stronger pitching effects from such control. This is supported by physical intuition and the numerical simulations performed previously. ${ }^{9}$ The lower range of phase differences, 140-180 deg, saw a large dropoff of thrust and lift generation. In fact, the bat came to a complete stop at one point with the phase difference at $140 \mathrm{deg}$. Therefore, we should not plan to use this range in control of flapping flight.

\begin{tabular}{|l|c|c|c|}
\hline & Flap Freq & $\Delta_{21}$ & $\Delta_{31}$ \\
\hline Experiment 1 & $2 \mathrm{~Hz}$ & $90 \mathrm{deg}$ & Varied \\
Experiment 2 & $2.5 \mathrm{~Hz}$ & $90 \mathrm{deg}$ & Varied \\
\hline
\end{tabular}

Table 1. Experiment Parameters
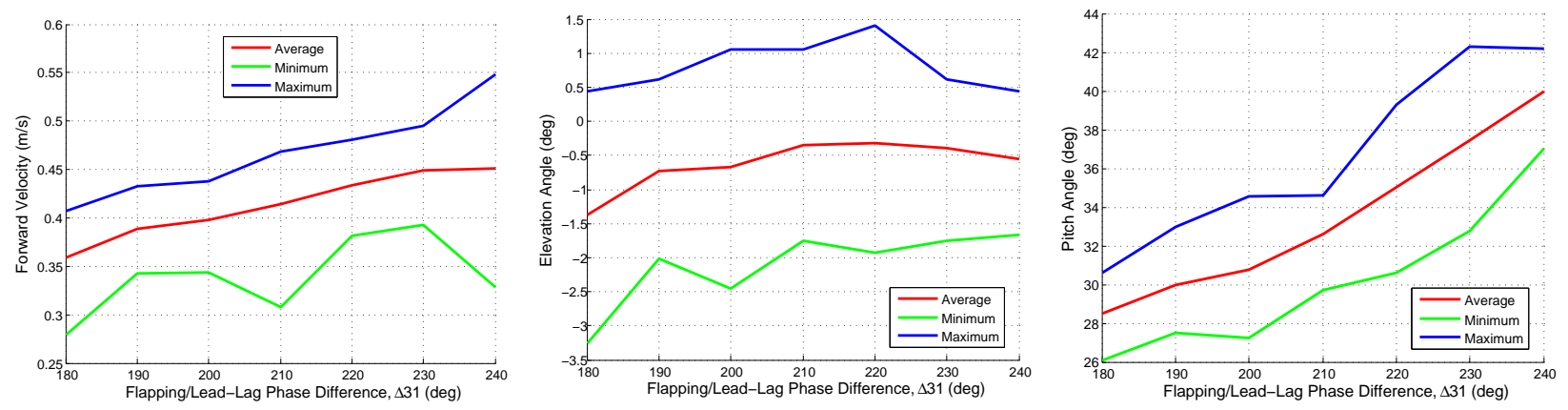

Figure 14. Open-loop pitch control via phase differences $(2.5 \mathrm{~Hz})$.

The second experiment repeated the same process at $2.5 \mathrm{~Hz}$ instead of $2 \mathrm{~Hz}$. Only one sweep through the values was performed. The results are plotted in figure 14 and support the same conclusion as the first experiment. Additionally, they preliminarily confirm the postulate that flapping frequency can be used as strong control of forward velocity and altitude. Finally, note that the authors are not concerned about the fact that all the relevant body pitch angles are all around 20-40 degrees. Adjustment of CG location can set the trim state as desired while control moments are created from phase differences.

\section{Conclusion}

The construction of the robotic bat and helicopter stand test bed will allow for a wider variety of experiments to be conducted in the field of flapping flight aerial vehicles. Previously, the robotic bat was mounted to a stationary stand and previous theories on dynamics and stability were unable to be thoroughly tested. While the range of motion is limited and the robotic bat is not truly flying, we can finally test the theory that controlling phase differences can achieve stable flight. In the near future we will conduct more experiments similar to those shown in this paper, and include more data for different flapping frequencies and amplitudes. We will also analyze the exact motion of the robotic bat's wings using a Vicon Motion Capture system and attempt to correct for mechanical coupling issues. Finally, we will develop a closed loop controller using the encoder data from the Quanser 3DOF Helicopter stand.

The test bed itself will need continuous improvements. Particularly, the mechanical structure of the robotic bat will be redesigned to further reduce mechanical coupling between wing motions and to improve the overall stability and rigidity of the structure when mounted on the helicopter stand. Different actuators may be used to control the wing motions, and for testing certain models it may be necessary to change the total number of actuators used. In the future, we would like to implement CPG's via field programmable gate arrays to truly demonstrate decentralized control with CPG's.

While progress is expected towards the development of autonomous flapping flight, current technological limitations are still far too great to accurately mimic biological flight systems and thus more research and development is required in several different areas of study in order for significant progress to be made in this area. 


\section{Acknowledgments}

This project was supported by the Air Force Office of Scientific Research (AFOSR) under the Young Investigator Award Program (Grant No. FA95500910089) monitored by Dr. W. Larkin. The authors would

like to thank the following students from the University of Illinois at Urbana-Champaign: Jonathan Yong, James Holtman, Andrew Meister, and Matthew Schipp.

\section{References}

${ }^{1}$ Deng, X., Schenato, L., Wu, W. C., and Sastry, S. S., "Flapping Flight for Biomimetic Robotic Insects: Part I-system Modeling," IEEE Trans. on Robotics, Vol. 22, No. 4, 2006, pp. 776-788.

${ }^{2}$ Ho, S., Nassef, H., Pornsinsirirak, N., Tai, Y.-C., and Ho, C.-M., "Unsteady Aerodynamics and Flow Control for Flapping Wing Flyers," Progress in Aerospace Sciences, Vol. 39, 2003.

${ }^{3}$ Birch, J. and Dickinson, M., "Spanwise Flow and the Attachment of the Leading-edge Vortex on Insect Wings," Nature, Vol. 412, Aug. 2001, pp. 729-733.

${ }^{4}$ S. Ho, H. Nassef, N. P. Y.-C. T. C.-M. H., "Flight Dynamics of Small Vehicles," Proceedings of the International Council of the Aeronautical Sciences (ICAS), Toronto, Canada, 2002, pp. pp. $551.1-551.10$.

${ }^{5}$ Pornsinsirirak, T., Lee, S.-W., Nassef, H., Gransmeyer, J., Tai, Y.-C., Ho, C.-M., and Keennon, M., "MEMS Wing Technology for a Battery-powered Ornithopter," Proceedings of the International Conferences on MEMS, Miyazaki, Japan, 2000, pp. 709-804.

${ }^{6}$ Jadhav, G., The Development of a Miniature Flexible Flapping Wing Mechanism for Use in a Robotic Air Vehicle, 2007, M.S. Thesis, Georgia Institute of Technology, Atlanta, GA.

${ }^{7}$ Wood, R. J., "The First Takeoff of a Biologically-inspired At-scale Robotic Insect," IEEE Transactions on Robotics, Vol. 24, No. 2, 2008, pp. 341-347.

${ }^{8}$ Swartz, S. M., Iriarte-Diaz, J., Riskin, D. K., Song, A., Tian, X., Willis, D. J., and Breuer, K. S., "Wing Structure and the Aerodynamic Basis of Flight in Bats," Proc. of the 45th AIAA Aerospace Science Meeting, Reno, NV, 2007.

${ }^{9}$ Chung, S.-J. and Dorothy, M., "Neurobiologically Inspired Control of Engineered Flapping Flight," AIAA Journal of Guidance, Control, and Dynamics, Vol. 33, No. 2, 2010, pp. 440-453.

${ }^{10}$ Azuma, A., The Biokinetics of Flying and Swimming, AIAA, 2nd ed., 2006.

${ }^{11}$ F Herrero-Carrón, F. B. R. and Varona, P., "Bio-inspired design strategies for central pattern generator control in modular robotics," Bioinsp. Biomim., Vol. 6, 2011.

${ }^{12}$ Chung, S.-J. and Slotine, J.-J. E., "Cooperative Robot Control and Concurrent Synchronization of Lagrangian Systems," IEEE Transactions on Robotics, Vol. 25, No. 3, 2009, pp. 686-700.

${ }^{13}$ Chung, S.-J., Dorothy, M., and Stoner, J. R., "Neurobiologically Inspired Control of Engineered Flapping Flight," AIAA Infotech at Aerospace and Unmanned Unlimited Conference and Exhibit, Seattle, WA, Apr. 2009, AIAA Paper 2009-1929.

14 "Q4 Hardware in the Loop (H.I.L) Board," http://www.quanser.com/net/industrial/Systems_and_Products/Prod_Q4_ Hardware.aspx, 2009.

${ }^{15}$ Quanser 3-DOF Helicopter Reference Manual, Quanser.

${ }^{16}$ Tian, X., Iriarte-Diaz, J., Middleton, K., Galvao, R., Israeli, E., Roemer, A., Sullivan, A., Song, A., Swartz, S., and Breuer, K., "Direct Measurements of the Kinematics and Dynamics of Bat Flight," Bioinspiration and Biomimetics, Vol. 1, 2006, S10-S19. 